\title{
MATHEMATICAL MODELLING OF CONSUMER CHOICE IN FASHION: POTENTIAL STUDENT PROJECTS

\author{
Anthony G Shannon
}

Faculty of Engineering \& IT, University of Technology, Sydney, NSW 2007, \&

Campion College, PO Box 3052, Toongabbie East, NSW 2146, Australia

Anthony.Shannon@uts.edu.au,t.shannon@campion.edu.au

\begin{abstract}
:
This paper suggests an unusual theme for undergraduate student projects. The future is now. Repackaging the past has historical value but it is not a preparation for the range and scope of the internet as a vast copying machine which can not only detect purchasing patterns but can adjust bargain prices to fit the buyer's calculated financial power and target them through intermediary subsidiaries in a universal online market. Quantitative techniques can now penetrate disciplines which once eschewed them. This paper looks at three such approaches in the context of consumer choice in fashion.
\end{abstract}

Keywords: Euclidean distance; fashion choice; dissonance; opinion space; intuitionistic fuzzy sets; matrices; vectors;

Academic Discipline And Sub-Disciplines: Education, Didactics of Mathematical Sciences

SUBJECT CLASSIFICATION: AMS Classification Numbers: 91D30, 01A80, 00 A09

TYPE (METHOD/APPROACH): Innovative student practical projects

\section{Council for Innovative Research}

Peer Review Research Publishing System

Journal: INTERNATIONAL JOURNAL OF RESEARCH IN EDUCATION METHODOLOGY

Vol. 6, No. 3

www.ijrem.com, ijremeditor@gmail.com 


\section{INTRODUCTION}

Mathematical modeling can is now applied in many areas that affect everyday lives [e.g.,19]. In an era of labour-saving device and information-overload, how come we seem to have fewer free hours and less disposable income [13]? Could it be that we are victims of the meta-servers which can not only extract data from our emails [14], but which can also target us with 'bargains' focused on our spending history and vulnerability [3]? Should the professional development of our students prepare them more for how digital systems both gather and repackage data?

Quantitative techniques now penetrate disciplines which once eschewed them. This paper looks at one such model in the context of consumer choice in fashion. "Will she ever wear the same dress twice as an adult? Will she recycle clothes into new objects, or wash them as we do today: At some point in her life I suspect laundry will become obsolete" [16].

What follows is an extension and slight modification of the models proposed by Miller et al [15], Groeber et al [11], and Temponi et al [20], who have developed mathematical models of the theoretical framework of consumer choice and social influence. Individual attraction and aversion for fashions are integrated into a societal-level model expressed in difference and differential equations with clear assumptions. This does not pretend for a moment to get into hypertext and networked media, but it is a pointer to the new numeracy. It is appropriate at a time when people with much formal education can be found who boast about little mathematics they know and who are repelled by symbols instead of realising that they are a tool of thought [12].

\section{BACKGROUND}

For many years now design software has made it possible to order clothes online [1]. Whether one would want to is a separate vexed issue [7]. The model expounded here builds on the fashion decision process of an individual. It is assumed, for instance, that this process is unidirectional. This is done for ease of exposition, though it is recognised that this process can become quite sophisticated [18].

An individual forms an impression of which style in a given range is attractive (or appropriate) for them and which is repulsive (or inappropriate) for them for his or her self-identity in a given place at a given time. We can use symbols as a short-hand device to represent the individuals in our sample so that the adoption of a style by an individual $i$ at time $t$ can be represented by $x_{i, t}$, so that if we have 5 individuals under consideration then their opinion profile is

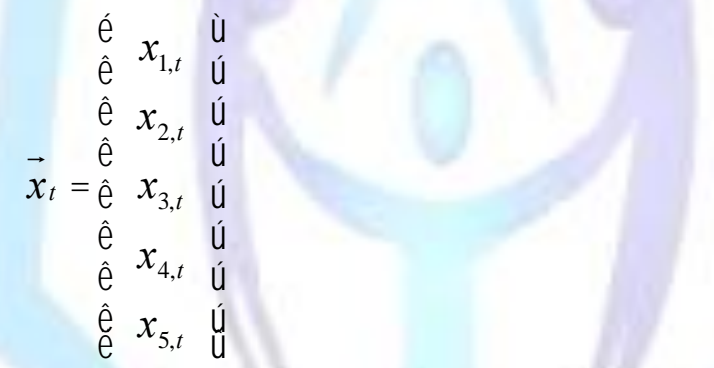

which can be transposed for later notational convenience into the form

$$
\vec{x}_{t}=x_{1, t}, \quad x_{2, t}, \quad x_{3, t}, \quad x_{4, t}, \quad x_{5, t}
$$

to represent the adoptions of style by the 5 members of our sample. More generally, for any number of individuals, $n,(2.2)$ can be re-written as

$$
\vec{x}_{t}=\quad x_{1, t}, \quad x_{2, t}, \quad \ldots \quad, \quad x_{n, t}
$$

For instance, consumer 3 in period 10 may select a style of tie with width a $75 \mathrm{~mm}$ as his 'utility-maximising style', so that in this case we could represent this as $x_{3,10}=75(\mathrm{~mm})$. For the (metric) opinion space $X$, the (Euclidean) distance function $d: X \times X \rightarrow R$ has the usual properties, namely,

- $\quad d(x, y)=0 \Leftrightarrow x=y$ (iff the opinions are equal in some sense);

- $\quad d(x, y)=d(y, x)$ (the order of the opinions is immaterial);

- $\quad d(x, y) \leq d(x, z)+d(z, y)$ (when there are three opinions to relate).

Distance is related to opinion forming in a cultural environment where there is a level of discomfort sensed because of different opinions. The dissonance of person $i$ at time $t$ with opinion $x_{i, t}$ depends on opinion differences with others in is set. Thus the size of the dissonance of persons $i$ and $j$ at time $t$ can be represented by $f^{-1}\left(d\left(x_{i, t}, x_{j, t}\right)\right)$ in which the negative superscript indicates that this function is intended to be minimised [9]. This magnitude may depend on the weight, 
$w_{i, j}$, of the relation between $i$ and $j$ so that the total dissonance function of person $i$ at time $t$ in this set can be represented by

$$
u_{i, t}^{-1}\left(x_{1, t}, x_{2, t}, \ldots, x_{n, t}\right)=\sum_{j \in X} w_{i j} f^{-1}\left(d\left(x_{i, t}, x_{j, t}\right)\right)
$$

if it is additively separable. At this stage we are merely attempting to see notation as a convenient tool of thought [12]. There are various models of opinion formation of fashion consensus to which the interested reader can refer [10]; we shall consider one such [15].

\section{SETTING UP A MODEL}

It is assumed that individual $i$ in time period $t$ can estimate which styles he or she finds attractive, $a_{i, t}$, and which repulsive, $r_{i, t}$. For instance, individual 3 might be attracted to $x_{1,1}$ and $x_{2,1}$, with the former twice as attractive as the latter in time period 1. People are often more willing to be forced to choose two or three preferences rather than forced to rank five or more. This can be represented as

$$
\begin{aligned}
a_{3,2} & =\frac{2}{3} x_{1,1}+\frac{1}{3} x_{2,1} \\
& =\frac{1}{3}\left(2 x_{1,1}+x_{2,1}\right) \\
& =\left[\begin{array}{lllll}
\frac{2}{3} & \frac{1}{3} & 0 & 0 & 0
\end{array}\right]\left[\begin{array}{llllll}
x_{1,1} & x_{2,1} & x_{3,1} & x_{4,1} & x_{5,1}
\end{array}\right]^{T}
\end{aligned}
$$

Similarly, this person might find the styles of $x_{4}$ and $x_{5}$ equally repulsive in the same time period, so that

$$
\begin{aligned}
r_{3,2} & =\frac{1}{2} x_{4,1}+\frac{1}{2} x_{5,1} \\
& =\frac{1}{2}\left(x_{4,1}+x_{5,1}\right) \\
& =\left[\begin{array}{lllll}
0 & 0 & 0 & \frac{1}{2} & \frac{1}{2}
\end{array}\right]\left[\begin{array}{lllll}
x_{1,1} & x_{2,1} & x_{3,1} & x_{4,1} & x_{5,1}
\end{array}\right]^{T} .
\end{aligned}
$$

All 5 consumers in our samples at all 5 time periods might then be represented as in Figures 1 and 2in which the columns represent time periods, $t$, and the rows represent individual consumers, $i$. In Table 1, the fractions have been related to 12 and in Table 2 to 10. (The former has more factors, but people are generally more comfortable with the latter.)

Table 1. Attractions

Table 2. Repulsions

\begin{tabular}{|c|c|c|c|c|c|}
\hline$A$ & 1 & 2 & 3 & 4 & 5 \\
\hline 1 & 0 & 8 & 4 & 0 & 0 \\
\hline 2 & 2 & 0 & 8 & 0 & 2 \\
\hline 3 & 0 & 2 & 0 & 10 & 0 \\
\hline 4 & 0 & 2 & 0 & 0 & 10 \\
\hline 5 & 10 & 0 & 0 & 2 & 0 \\
\hline
\end{tabular}

\begin{tabular}{|c|c|c|c|c|c|}
\hline$R$ & 1 & 2 & 3 & 4 & 5 \\
\hline 1 & 0 & 3 & 0 & 4 & 3 \\
\hline 2 & 6 & 0 & 0 & 4 & 0 \\
\hline 3 & 0 & 5 & 0 & 0 & 5 \\
\hline 4 & 3 & 0 & 5 & 0 & 2 \\
\hline 5 & 1 & 2 & 5 & 2 & 0 \\
\hline
\end{tabular}

Mathematically, they can be expressed as column vectors:

$$
\vec{a}_{t}=\left[\begin{array}{lllll}
a_{1, t}, & a_{2, t}, \quad \ldots & a_{n, t}
\end{array}\right]^{T} \text { and } \vec{r}_{t}=r_{1, t}, \quad r_{2, t}, \quad \ldots \quad, \quad r_{n, t}
$$

and matrices

$$
A=\left[\vec{a}_{1}, \vec{a}_{2, \ldots x}, \vec{a}_{n}\right] \text { and } R=\left[\vec{r}_{1}, \vec{r}_{2}, \ldots, \vec{r}_{n}\right] \text {, }
$$

exemplified in our hypothetical case from Tables 1 and 2 by Table 3 in which the integers in the cells have been converted to decimals as explained above. 
Table 3. Matrix expressions of Tables 1 and 2

$$
A=\left[\begin{array}{ccccc}
0 & \frac{2}{3} & \frac{1}{3} & 0 & 0 \\
\frac{1}{6} & 0 & \frac{2}{3} & 0 & \frac{1}{6} \\
0 & \frac{1}{6} & 0 & \frac{5}{6} & 0 \\
0 & \frac{1}{6} & 0 & 0 & \frac{5}{6} \\
\frac{5}{6} & 0 & 0 & \frac{1}{6} & 0
\end{array}\right], \quad R=\left[\begin{array}{ccccc}
0 & 0.3 & 0 & 0.4 & 0.3 \\
0.6 & 0 & 0 & 0.4 & 0 \\
0 & 0.5 & 0 & 0 & 0.5 \\
0.3 & 0 & 0.5 & 0 & 0.2 \\
0.1 & 0.2 & 0.5 & 0.2 & 0
\end{array}\right] .
$$

The row and column sums equal unity. This is not essential but although it makes comparisons more obvious, it neglects the influence of a given individual on the fashions of the time in a given society [11] - given by the row sums. Doing this for a small number of participants is much less difficult than a Sudoku puzzle; for large numbers a spreadsheet can be used for this and for later calculations [2]. The matrices $A$ and $B$ contain the relative weights: quantifications of relative attraction or disdain.

We now introduce some parameters to further quantify and clarify the concerns of consumer $i$

- $\quad c_{a, i}$ for appropriateness (for self-identity),

- $\quad c_{r, i}$ for inappropriateness (for self-identity),

- $c_{c, i}$ for change from one period to another,

so that we can produce an additively separable quadratic function for 'utility' as seen by this consumer $i$. In this we take squared differences, because if linear differences were used a large negative difference might merely cancel out a large positive difference so that the sum would not reflect is utility function which we aim to maximise. We also introduce a constant of proportionality for each consumer, $U_{i}$, which could be found when actual numbers are inserted. The utility sum for consumer $i$ is then

$$
U_{i} x_{i, t}=-c_{a, i}\left(x_{i, t}-a_{i, t}\right)^{2}+c_{r, i}\left(x_{i, t}-r_{i, t}\right)^{2}-c_{c, i}\left(x_{i, t}-x_{i, t-1}\right)^{2} .
$$

The conditions for utility maximisation are found when the first derivative is zero and the corresponding second derivative is negative; that is,

$$
\left(\frac{d}{d x_{i, t}}\right) U_{i} x_{i, t}=-2\left(c_{a, i}\left(x_{i, t}-a_{i, t}\right)-c_{r, i}\left(x_{i, t}-r_{i, t}\right)+c_{c, i}\left(x_{i, t}-x_{i, t-1}\right)\right)=0
$$

and

$$
\left(\frac{d}{d x_{i, t}}\right)^{2} U_{i} x_{i, t}=-2\left(c_{a, i}-c_{r, i}+c_{c, i}\right)<0
$$

That is, the utility maximising style $x_{i, t}$ for individual $i$ in time period $t$ is

$$
x_{i, t}=\frac{c_{a, i} a_{i, t}-c_{r, i} r_{i, t}+c_{c, i} x_{i, t-1}}{c_{a, i}-c_{r, i}+c_{c, i}}
$$

for $i=1,2, \ldots, n$. For all $n$ individuals at the same time

\section{INTUITIONISTIC FUZZY MODELLING}

It is recognised that there can be significant feedback among the steps outlined above, so that the scheme could be enhanced with generalized nets [4], particularly if they incorporate intuitionistic fuzzy logic and vary the nature of the opinion space $[5,6,15]$ in which feedback operates through attraction and repulsion of former styles as well as current fashions. There is also the related, but separate factor, of how the styles of one country influence the styles in another country (positively or negatively). An outline of key steps in this approach now follow [16].

Let $E$ be a fixed set. An intuitionistic fuzzy set (IFS) $A$ in $E$ is an object of the following form

$$
A=\left\{\left\langle x, \mu_{A}(x), v_{A}(x)\right\rangle \mid x \in E\right\}
$$


in which the functions $\mu_{A}: E \rightarrow[0,1], v_{A}: E \rightarrow[0,1]$ respectively define the degree of membership and the degree of non-membership of the element $x \in E$, and for every $x \in E$ :

$$
0 \leq \mu_{A}(x)+v_{A}(x) \leq 1 .
$$

For every two IFSs $A$ and $B$ many logical operations, relations and operators have been defined [5]. For the universe $E$ let the figure $F$ be given in the Euclidean plane and with Cartesian coordinates (Figure 1).

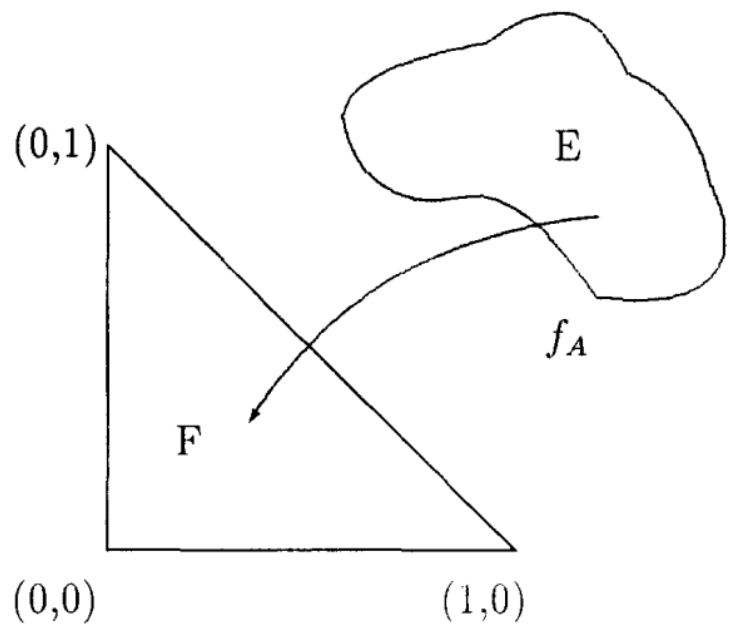

Fig. 1.

Let $A \subset E$ be a fixed set so that we can construct a function $f_{A}: E \rightarrow F$ such that if $x \in E$, then $p=f_{A}(x) \in F$, and the point $p$ has coordinates $<a, b>$ for which $a=\mu_{A}(x)$, and $b=v_{A}(x)$. We then introduce operators $C$ and / with geometrical interpretations given in Figures 2 and 3 respectively.

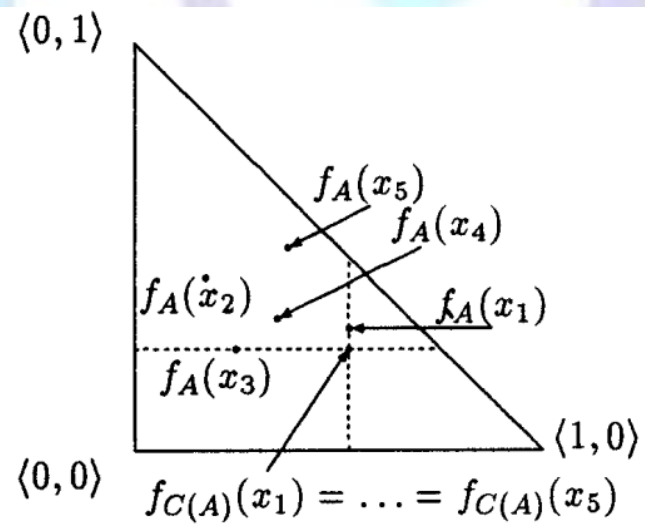

Fig 2. 


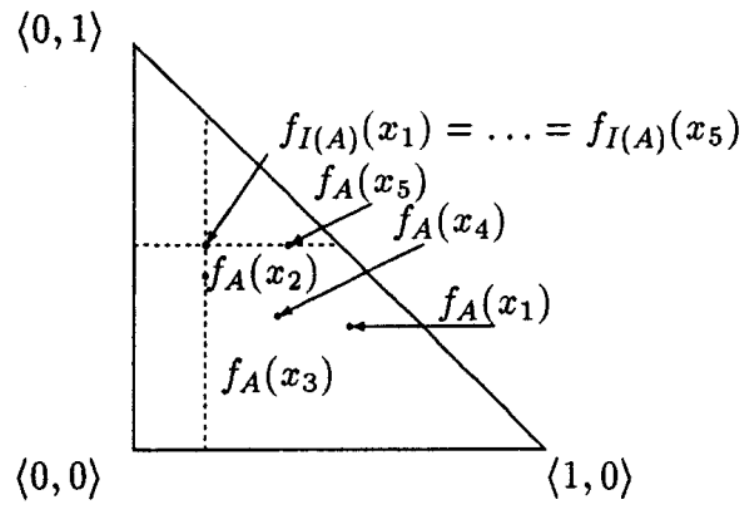

Fig 3.

We can use the operators $C$ and $/$ to construct an area in each interpretational triangle. This area will contain all points which are interpretations of the given IFS $A$. We define a weight operator $W$, the weight-centre of these points. This becomes a criterion for the correctness (validity) of the elements of $A$ [17]. In a simplified model the various criteria can be reduced to two possibilities, namely:

- if the point $W(A)$ is nearly to the left upper sides of the area, then a larger number of elements of the IFS $A$ have 'bad' values for their choice, but

- if the point $W(A)$ is nearly to the right lower sides of the area, then a larger number of elements of the IFS $A$ have 'good' values for their choice.

\section{CONCLUDING COMMENTS}

Those who wish for an explanation of the historical framework for understanding the fashion process should read [11] and follow some of its references. For insights into fashion media in general, and fashion blogging in particular, see [8,9]. Here we have merely outlined some of the issues in consumer choice in fashion, where an individual's fashion choices are associated with self-identity, attraction to esteemed groups, desire to be distanced from perceived repulsive styles, and attitude towards change.

\section{REFERENCES}

1) Aldruch, Winifred (ed.). 1992. CAD in Clothing and Textiles. Oxford: BSP Professional Books.

2) Arganbright, Deane. 1993. Practical Handbook of Spreadsheet Curves and Geometric Constructions. Boca Raton, FL: CRC Press.

3) Ariely, Dan. 2008. Predictably Irrational: The Hidden Forces that Shape our Decisions. New York: HarperCollins.

4) Atanassov, Krassimir T. 1991. Generalized Nets. Singapore, New Jersey/London: World Scientific.

5) Atanassov, K.T. 1999. Intuitionistic Fuzzy Sets: Theory and Applications. Heidelberg/New York: Physica-Verlag.

6) Atanassov, K.T., Shannon, A.G. 1998. A Note on Intuitionistic Fuzzy Logics. Acta Philosophica. 7: 121-125.

7) Bianco, Anthony. 2007. Wal-Mart: The Bully of Bentonville - How the High Cost of Everyday Prices Is Hurting America. New York: Currency Doubleday.

8) Findlay, R. 2012. At One Remove from Reality: Style Blogger and Outfit Posts. Australasian Journal of Popular Culture. 1 (2): 1897-208.

9) Findlay, Rosie. 2014. O HAI GUYZ: Between Personal Style Bloggers, Their readers and Modern Fashion. Doctor of Philosophy Thesis, Faculty of Arts and Social Sciences, The University of Sydney. 
10) Friedkin, N., Johnsen, E. 1990. Social Influences and Opinions. Journal of Mathematical Sociology. 15: $193-205$.

11) Groeber, Patrick, Lorenz, Jan, Schweitzer, Frank. 2014. Dissonance Minimization as a Microfoundation of Social Influence in Models of Opinion Formation. Journal of Mathematical Sociology. 38: 147-174.

12) Iverson, Kenneth. E. 1980. Notation as a Tool of Thought. Communications of the Association of Computing Machinery. 23 (8): 444-465.

13) Kahneman, Daniel. 2011. Thinking, Fast and Slow. New York: Farrar, Strauss and Giroux.

14) Lanier, Jaron. 2013. Who Owns the Future? New York: Simon and Schuster.

15) Miller, Christopher M., McIntyre, Shelby H., Mantrala, Murali, K. 1993. Toward Formalizing Fashion Theory. Journal of Marketing Research. 30(2): 142-158.

16) Miller, Christopher M., Smith, Stephen A., McIntyre, Shelby H., Achabal, Dale D. 2010. Optimizing and Evaluating Retail Assortments for Infrequently Purchased Products. Journal of Retailing. 86(2): 159-171.

17) Mordeson, John N., Clark, Terry D., Albert, Karen. 2014. Factorization of intuitionistic fuzzy preference relations. New Mathematics and Natural Computation. 10 (1): DOI: 10.1142/S179300571450001X.

18) Shannon. A.G. 1982. Note on an algebraic structure for a concept space. Journal of Structural Learning. 7: 143-150.

19) Shannon, Anthony G. 2011. Reflections on Some Mathematical Modelling in Endocrinology. International Journal Bioautomation. 15 (3): 183-200.

20) Temponi, Cecilia, Shannon, Anthony, Atanassov, Krassimir, Ban, Adrian. 1999. An idea for an intuitionistic fuzzy approach to decision making. Notes on Intuitionistic Fuzzy Sets. 5 (3): 6-10.

\section{AUTHOR'S BIOGRAPHY WITH PHOTO}

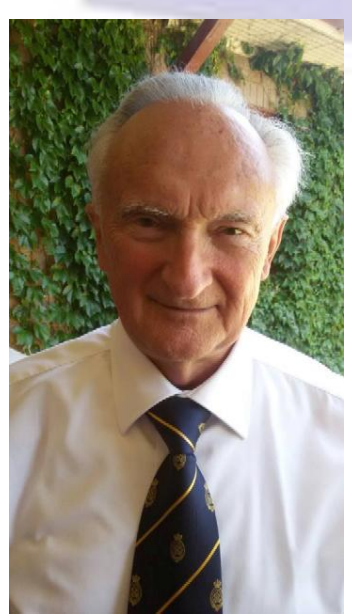

Professor A. G. (Tony) Shannon AM is an Emeritus Professor of the University of Technology, Sydney, where he was Foundation Dean of the UTS Graduate Research School and Professor of Applied Mathematics, and where he is currently doing research in the Centre for Health Technologies within the Faculty of Engineering and Information Technology.

He holds the doctoral degrees of Ph.D., Ed.D. and D.Sc. He is co-author of numerous books and articles in medicine, mathematics and education. His research interests are in the philosophy of education, number theory, and epidemiology, particularly through the application of generalized nets and intuitionistic fuzzy logic. He has taught and mentored at all levels from primary school to post-doctoral.

Professor Shannon is a Fellow of several professional societies. He is presently Registrar of Campion College, a liberal arts degree granting institution in Sydney. In June 1987 he was appointed a Member of the Order of Australia (AM) for services to education. He enjoys reading, walking, theatre, number theory, and thoroughbred racing. 